\title{
Bacillus sphaericus: biocontrolador de vectores que producen malaria, fiebre amarilla y dengue
}

\section{Sara Esmeralda Gómez Romero', Cindy Zulay Hernández Rodríguez', Lucía Constanza Corrales Ramírez MSc ${ }^{2}$}

1. Estudiantes del Programa de Bacteriología y Laboratorio Clínico de la Universidad Colegio Mayor de Cundinamarca

2. Docente Investigador Universidad Colegio Mayor de Cundinamarca

Correspondencia: Icorralesr07@gmail.com

Recibido: 11-06-09 / Aceptado: 21 -10-09

\section{Resumen}

El aumento en la incidencia de enfermedades de interés en salud pública como malaria, dengue y fiebre amarilla asociado a los efectos secundarios del uso de plaguicidas químicos y la resistencia generada, ha impulsado a la búsqueda de nuevas alternativas para la erradicación de sus vectores. Una opción viable como bacteria entomopatógena es el Bacillus sphaericus, descrita por primera vez en 1904 por Neide y Meyer, quienes descubrieron una bacteria formadora de esporas, que existe en la naturaleza y que crece fácilmente tanto in vitro como en el cadáver de las larvas.

Su actividad larvicida se debe a la producción de una inclusión cristalina denominada toxina binaria A y $B$ de 4I,9 y 5 I ,4 kDa, respectivamente, las cuales, al ser liberadas en el intestino de los insectos susceptibles en su fase larvaria y tras ser solubilizada por el pH alcalino en la porción media del intestino, activan las proteasas causando deshidratación y muerte a los vectores. Es considerada como un biolarvicida altamente potencial y eficaz para el control biológico de Anopheles spp., Aedes spp. y Culex spp. Comercialmente puede producirse por fermentación sumergida a pequeña escala con materiales proteínicos de desecho. Este producto ha sido utilizado y registrado en numerosos países de América Latina, donde se han obtenido buenos resultados después de su aplicación.

Palabras clave: Bacillus sphaericus, biocontrolador, biolarvicida, toxina binaria Bin A y Bin B.

\section{Abstract \\ Bacillus sphaericus: vector control strategies that produce malaria, yellow fever, and dengue}

The increased incidence of diseases of public health such as malaria, dengue and yellow fever associated to side effects of the use of chemical pesticides and the resistance generated has prompted the search for new alternatives for the elimination of its vectors. A viable option is the entomopathogenic bacteria Bacillus sphaericus, first described in 1904 by Neide and Meyer who discovered spore-forming bacteria that exists in nature and is easily grown in vitro and in the body of the larvae.

Its larvicidal activity is due to the production of a crystalline inclusion, called binary toxin $A$ and $B$ of 41.9 and $51.4 \mathrm{kDa}$, respectively, which, when released in the gut of susceptible insects in their larval 
stage and after be solubilized by the alkaline $\mathrm{pH}$ in the middle portion of the intestine, activate proteases causing dehydration and death of the vectors. It is considered a highly potential and effective larvicide for biological control of Anopheles spp., Aedes spp. and Culex spp. It can be produced commercially by submerged fermentation with small-scale waste protein material. This product has been used and registered in many Latin American countries, where good results were obtained after application.

Keywords: Bacillus sphaericus, binary toxin Bin A and Bin B, biocontrol, larvicide.

\section{Introducción}

En Colombia las enfermedades de alto interés epidemiológico como la malaria, dengue y fiebre amarilla, se han convertido en un problema de salud pública a nivel nacional. Según el informe epidemiológico que pública el Instituto Nacional de Salud (INS) durante diciembre 28 de 2007 a enero 3 de 2009, se reporta una mortalidad de 12 y 14 casos por dengue y malaria, respectivamente. Igualmente los casos confirmados de dengue corresponden a 26795, que en su mayoría fueron presentados en los departamentos de Norte de Santander, Santander, Cauca, Casanare, Meta, Valle del Cauca, Tolima y Bolívar, principalmente. De malaria se reportaron 6398 casos por Plasmodium Falciparum, 14 casos por Plasmodium Malariae, 569 casos de malaria mixta y 25681 casos por Plasmodium vivax, que corresponden en su mayoría a los departamentos de Córdoba, Antioquia, Meta, Guaviare, Putumayo y Valle del Cauca. De fiebre amarilla se reportaron dos casos confirmados, en el departamento de Caquetá (1).

Los datos descritos originan la necesidad de realizar un análisis profundo sobre las causas que influyen en la persistencia y aumento de éstas enfermedades en nuestro país. Por lo tanto, es inminente la búsqueda de nuevas alternativas de control que disminuyan la morbi-mortalidad, ya que a diario miles de personas enferman y otras mueren a causa de ellas. La observación desde la vigilancia de salud pública ha detectado que factores como el desarrollo de monocultivos y la utilización indiscriminada de pesticidas de amplio espectro y alta persistencia han precipitado el incremento de plagas y enfermedades, cuyos vectores a su vez generan mecanismos de resistencia que conllevan a que su erradicación sea más difícil (2).
Este contexto generó en los científicos interesados en el tema de salud pública, la necesidad de realizar estudios que planteen soluciones a la situación de emergencia de estas enfermedades endémicas. Una opción que ha tomado fuerza es la implementación de nuevas alternativas para el control de los vectores, con el objetivo de reemplazar los insecticidas químicos por productos biológicos, cuya acción consiste fundamentalmente en la manipulación del sistema de regulación natural por medio del control biológico que puede definirse como el "uso de organismos naturales o modificados, genes o productos de genes, para reducir los efectos de organismos indeseados (vectores) y favorecer a organismos deseables como cultivos vegetales, animales o microorganismos, sin producir efectos adversos para el hombre y el medio ambiente" (3). Así que el interés se volcó hacia las bacterias entomopatógenas (4) que resultan una opción viable en el control biológico de vectores como Anopheles spp., Aedes spp y Culex spp, (5) de gran importancia epidemiológica en la transmisión de enfermedades en las zonas tropicales de nuestro país, encontrando dentro de ellas el "Bacillus sphaericus".

\section{Origen del Bacillus sphaericus}

Fue descrito por primera vez en el año de 1904 por Neide y Meyer $(6,7)$, quienes descubrieron una bacteria que por sus características de esporulación la llamaron Bacillus sphaericus. En 1965, investigadores de California, a partir de una larva enferma del mosquito Culiseta incidens, aislaron la primera cepa de esta bacteria (8). Desde entonces en diferentes partes del mundo se han aislado varias cepas de esta bacteria que son patógenas para las larvas de mosquitos, encontrando la cepa 1593 aislada de larvas enfermas de Culex quinquefasiatus recogidas en drenajes 
contaminados en Rawasari, Jakarta, Indonesia, en junio de 1974. Actualmente, la cepa que se utiliza con mayor aceptación en Centro América y en el mundo, es Bacillus sphaericus 2362, la cual fue aislada por J. Weiser en Nigeria en la década de 1980 (9).

\section{Características macroscópicas, microscópicas y bioquímicas}

Bacillus sphaericus es un bacilo Gram-positivo que mide 0.61 a 1 micra de ancho y 1.5 a 5 micras de largo, con bordes redondeados, posee una espora terminal (producida en condiciones adversas) que deforma el bacilo, dándole un aspecto de raqueta; macroscópicamente puede observarse que produce colonias beta hemolíticas, color blanco crema traslucido y bordes regulares (10). Este microorganismo tiene metabolismo aerobio, se desarrolla en amplios rangos de temperatura: máxima entre $30^{\circ} \mathrm{C}$ y $45^{\circ} \mathrm{C}$ y mínima de $5^{\circ} \mathrm{C}$ a $15^{\circ} \mathrm{C}$, es catalasa positiva, no fermenta la glucosa, no reduce nitratos, es fenilalanina positivo. Sensible a bajas concentraciones de penicilina y tetraciclina y presenta resistencia al cloramfenicol y a la estreptomicina (11).

\section{Características genéticas}

La secuenciación revela que el genoma completo de esta bacteria entomopatógena está compuesto por un cromosoma circular de $4639821 \mathrm{pb}$, con un promedio en el contenido de G-C de 37,29\% y un plásmido replicón (llamado pBsph) de 177.642 $\mathrm{pb}$, con un promedio en el contenido de G-C de $33,10 \%$; con 4786 y 186 posibles secuencias de codificación de proteínas, respectivamente (12). Por estudios de homología del DNA, se dan cinco grupos genéticamente distintos (I - V); cada uno de ellos probablemente correspondiente a una especie diferente (13). El Grupo II se divide en los grupos IIA y IIB, a los cuales están asociados nueve serotipos $(\mathrm{H} 1, \mathrm{H} 2$, H3, H5, H6, H9, H25, H26 y H48) $(8,11)$.

\section{Inclusión cristalina}

En las primeras fases del proceso de esporulación el Bacillus sphaericus produce un cristal que contiene una proteína con gran poder tóxico frente a las larvas de mosquitos. Esta proteína es considerada como un biolarvicida altamente eficaz para el control biológico de Anopheles spp., Aedes spp y Culex spp. (14), que se compone de dos polipéptidos codificados por separado: $\operatorname{BinA}(41,9 \mathrm{kDa})$ y $\operatorname{BinB}(51,4 \mathrm{kDa})(15)$. Las proteínas de la toxina binaria son sintetizadas en cantidades equimolares, durante las fases tempranas de la esporulación (16), formando las inclusiones cristalinas en la paraespora (17) y es codificada por dos genes (Bin A y Bin B) $(18,20)$, que han sido clonados y secuenciados de su material genético, como polipéptidos que se requieren para ejercer el efecto tóxico (19).

Esta toxina presenta diferentes rangos de especificidad de acuerdo al hospedero, ya que es principalmente tóxica para las larvas de Culex spp. (21-24) y Anopheles $s p p$. $(22,24)$ y en menor potencia para Aedes spp. (23). La existencia de cepas con mayor o menor toxicidad está relacionada con la expresión genética de las proteínas que conforman la toxina $(25,26)$. Se ha demostrado que BinB es el componente primario obligatorio que actúa como ligando, dirige y localiza a BinA en las células blanco (27). En el caso de Culex spp. BinB - BinA juegan un papel importante de unión al receptor para ligarse a las células intestinales, el proceso que sigue es la internalización de la toxina en la célula intestinal (28).

Cuando las larvas de mosquitos ingieren las esporas y los cristales proteicos, éstos se disuelven en medio alcalino, con la ocurrencia de proteólisis (29), liberándose en el medio fragmentos tóxicos que son reconocidos por receptores específicos que se encuentran en las células epiteliales del intestino, lo que induce a la formación de poros en las membranas de la porción distal de las microvellosidades de la mucosa del ciego y del intestino medio. Esta unión y la acción de la toxina, además provocan en la membrana de estas células cambios concomitantes en los organelos citoplasmáticos que incluyen la desintegración del retículo endoplasmático, condensación y tumefacción de las mitocondrias y dilatación del espacio perinuclear. Como consecuencia de lo anterior ocurre hipertrofia y ruptura de las células del intestino medio y ciego, un desbalance iónico que lleva a toxemia y bacteremia cuyo resultado es la muerte de la larva (30). 
Observaciones de las células epiteliales del intestino de la larva han mostrado cambios morfológicos. Estudios fisiológicos del Culex quinquefasciatus han evidenciado que la toxina binaria también actúa sobre la membrana celular en la cual puede causar poros como los producidos por el Bacillus thuringiensis que conllevan a la muerte celular (31-33).

\section{Enzimas}

Bacillus sphaericus es un alcalófilo obligado, que produce gran cantidad de proteasa alcalina extracelular, la cual es una enzima altamente empleada en la industria farmacéutica y alimentaria $(34,35)$. En el Himalaya han encontrado más aplicaciones relacionadas con esta enzima, por cuanto presenta buenos resultados como aditivo a detergentes debido a la compatibilidad que muestra con el cloro $(36,37)$, y a la recuperación de radiografías usadas. En la purificación de dos nuevas proteasas extracelulares fue encontrada la serin proteasa (38) con una actividad proteasa del $98 \%$, por lo que nuevos estudios se han encaminado a la biosíntesis de esta enzima a gran escala, su producción es dependiente de la fuente de nitrógeno del medio, por lo cual se han utilizado extractos de levadura, peptona y glucosa como alternativas nutricionales que han mostrado buenos resultados $(39,40)$.

Con el fin de conocer más sobre la actividad proteasa, se ha recurrido a realizar estudios cinéticos, los cuales han demostrado que la síntesis y secreción de esta enzima se produce en la fase de crecimiento bacteriano, alcanzando su mayor producción de dos a tres horas después de iniciados los procesos de fermentación, requiriendo concentraciones mínimas de substrato y nutrientes como glucosa, debido a que el incremento en la concentración de estas no tiene efecto sobre el crecimiento (37).

Nuevos estudios han demostrado que el poder tóxico no está limitado al poder biolarvicida sino que presenta otras toxinas, como la sphaericolisina de 53 $\mathrm{KDa}$ (41), que tiene efecto sobre dictiópteros de la clase insecta como las cucarachas, al unirse por su $\mathrm{N}$ - terminal al colesterol de las membranas formando poros que paralizan rápidamente el sistema nervioso central y otros tejidos recubiertos de colesterol y que además producen hemólisis en los eritrocitos causando la muerte al insecto (42).

\section{Producción industrial}

La biomasa de Bacillus sphaericus puede obtenerse por fermentación sumergida, producción en pequeña escala a partir de materiales proteínicos de desecho $y$ al ser una bacteria aerobia estricta requiere una alta aireación para su desarrollo. A $42^{\circ} \mathrm{C}$ se reduce la formación de toxina, sin afectar el crecimiento y la esporogénesis del microorganismo. En algunos casos el crecimiento y potencia de la cepa se logra bien en medio de solución de sales minerales (SSM) que tiene vitaminas, aminoácidos, glicerol y sales minerales y el RAW medio industrial que contiene harina de pescado, aceite de soya, vitaminas y cloruro de calcio (40).

Con el interés de obtener un mayor rendimiento en la acción biológica que cumplen estos microorganismos y minimizar los costos de producción industrial (39), puesto que los métodos empleados de fermentación resultan muy costosos, se han realizado estudios que buscan nuevas alternativas, generando la producción de nuevos medios de cultivo a expensas de residuos cerveceros y levaduras, los cuales han demostrado que promueven el crecimiento y la esporulación de la bacteria (43). También se ha considerado el empleo de materiales de desecho bio-orgánicos (plumas de pollo) como otra alternativa al proveer gran cantidad de $\beta$-queratina para el desarrollo de la bacteria (44). Se han realizado ensayos con cinco medios de cultivo que contienen semillas de legumbres, sangre de vaca seca y sales minerales. A partir de estos nuevos medios de cultivo se ha obtenido una mayor producción de las toxinas del insecticida específicamente contra especies de Culex, Anopheles y Aedes (45). Otros medios producidos a partir de comida para peces, fríjol de soya y maíz han reportado eficiencia para la producción en masa (46-48), al igual que la utilización de papas en un medio de fermentación (49).

\section{Aplicación}

El Bacillus sphaericus se aplica típicamente a aguas con alto contenido orgánico donde viven las 
larvas de los mosquitos (50). Este microorganismo tiene el beneficio de presentar mayor persistencia en el ambiente, debido a su capacidad de reciclarse, reproducirse en los cadáveres de las larvas afectadas e infectar otras (51). Su uso no se permite en los depósitos que contienen agua potable ya que este microorganismo tiene eficacia en aguas ricas en materia orgánica. Las esporas de Bacillus sphaericus sobreviven bien en la naturaleza y parece que el reciclado ocurre en el suelo.

Estudios de la acción larvicida de las muestras ha demostrado que las de mayor profundidad tienen mayor actividad que las superficiales, ya que no se observa actividad larvicida después de una exposición a la luz directa y en habitats con poca actividad luminosa dan resultados residuales por más largo tiempo (4). Su acción patógena depende de factores como: dosis de aplicación, ingestión de la toxina por la larva, velocidad de alimentación de la larva, rapidez con la cual la bacteria cae al fondo del criadero y se vuelve inaccesible a los anofelinos, competencia con otras materias orgánicas, grado de contaminación del agua, presencia o ausencia de vegetación y de los hábitos alimentarios de los vectores. Ensayos de campo han demostrando la ausencia de efectos sobre invertebrados no blanco de este microorganismo como crustáceos, dípteros, langostinos, renacuajos, escarabajos, mosca de la fruta, mosca negra, polilla de harina, al igual que vertebrados presentes en lugares de aplicación de este biolarvicida (52).

Latinoamérica ha mostrado gran interés en la producción industrial y aplicación del biolarvicida, el Laboratorio Biológico Farmacéutico (LABIOFAM) de Cuba, ha desarrollado los protocolos para este fin. Este producto ha sido utilizado y registrado en numerosos países de la región, donde se han obtenido buenos resultados después de su aplicación. La fórmula se presenta en forma de suspensión acuosa, que contiene las esporas y los cristales tóxicos del mencionado bacilo, los cuales destruyen las larvas de los mosquitos después de las primeras $72 \mathrm{~h}$ (53).

En Nicaragua durante el año 1996, se reportan resultados exitosos después de la aplicación aérea y terrestre del biolarvicida en la costa del lago Xolotlan,
Managua, con un registro de efectividad hasta de $100 \%$ tres meses después del tratamiento biológico sobre larvas de Anopheles albimanus (54).

El producto también fue evaluado en criaderos urbanos y periurbanos en el municipio de Buenaventura, en el Valle del Cauca, Colombia, para el control de dos importantes especies vectores de malaria en la región: Anopheles albimanus y Anopheles nuñeztovari. Los resultados indican que el biolarvicida es altamente efectivo para controlar estas especies, y se ha comprobado además que la duración de la actividad es de por lo menos tres meses en los criaderos tratados (55).

Los resultados epidemiológicos obtenidos en Managua y en el departamento de Escuintla, coinciden con los reportados por los boletines epidemiológicos del Sistema Local de Asistencia en Salud (SILAIS) durante el año 1997, después de la aplicación del controlador biológico (GRISELESF) para vectores maláricos en ese país, reportándose una reducción en el $25 \%$ de los casos de malaria en relación con los ańos 1995 y 1996, en todo el territorio del SILAIS, Managua. Sin embargo, al tener en cuenta sólo las áreas de intervención del tratamiento biológico, fue posible apreciar una reducción promedio de $50 \%$ de los casos acumulados de la enfermedad respecto a los dos ańos anteriores (56).

En la República de Guatemala los resultados obtenidos durante el año 1998 fueron satisfactorios, así como los logrados durante ańos anteriores en distintos países de la región después de la utilización del control biológico de vectores maláricos con Bacillus sphaericus cepa 2362. Esto demuestra la posibilidad de incorporar este método como parte de los programas de control integrado de vectores maláricos, en correspondencia con lo recomendado por la OPS/OMS (51).

Finalmente, se puede decir que este bacilo posee ventajas frente a otro tipo de microorganismos con capacidad biocontroladora como el Bacillus thuringiensis, ya que posee mayor efectividad y persistencia en el ambiente $(57,58)$. De igual forma el aislamiento de microorganismos como el Bacillus sphaericus dentro de territorio colombiano contribuye 
al manejo de enfermedades de interés en salud pública como malaria, fiebre amarilla y dengue, ya que al ser estas cepas nativas ofrecen mejores condiciones de adaptación y eficacia.

Sin embargo, es necesario realizar a las cepas nativas del Bacillus sphaericus además de la identificación microscópica, macroscópica y bioquímica, la caracterización molecular para identificar los genes que codifican para sus proteínas tóxicas, en este caso la proteína larvicida (toxina binaria Bin A y Bin B), con el objeto de ratificar el poder tóxico y proporcionar un aporte eficaz en los programas de control epidemiológico de vectores, mediante el biocontrol, el cual garantiza mantener el equilibrio en el medio ambiente, al no producir efectos adversos en la naturaleza ni en el humano; así como el aporte a nivel científico que contribuye con campos de investigación en nuestro país.

\section{Referencias}

1. Sistema de Vigilancia en Salud Pública - SIVIGILA. Semana Epidemiológica 53 (Diciembre 28 de 2007 a Enero 3 de 2009) actualizado el 20 de Enero de 2009 y Acumulados del Año 2009.

2. Romero D, Rivera ME, Cazorla FM, de Vicente A, Pérez-García A. Efect of mycoparasitic fungi on the development of Sphaerotheca fusca in melon leaves. Mycol Res. 2003;107:64-71.

3. Organización Mundial de la Salud (OMS). Lucha Biológica Contra los Vectores de Enfermedades. Sexto Informe del Comité de Expertos de la OMS en Biología de los Vectores y Lucha Antivectorial. (1982). Serie de Informes Técnicos No. 679;39.

4. Charles J, Delécluse A, Nielsen-LeRoux. Entomopathogenic bacteria: from laboratory to field application. ed. Kluwer Academic Publisher. 2000 p. 253-273.

5. Regis L, Silva-Filha MH, Nielsen-LeRoux C, Charles JF.. Bacteriological larvicides of dipteran disease vectors. Trends Parasitol. 2001;8:377-380.

6. Colin R. Harwood, Bacillus: taxonomy. Biotechnology Handbooks: New York.1989;2;5-22

7. Kellen WR, Clark TB, Lindegren JE, Ho BC, Rogoff MH, Singer S. Bacillus sphaericus Neide as a pathogen of mosquitoes. J Invertebr Pathol. 1965;7:442-448.

8. Organización Mundial de la Salud (OMS). Información técnica sobre el agente de control biológico. Bacillus sphericus cepa 1593. 1984. Serie Ecológica 10.

9. Guía para la implementación y demostración de alternativas sostenibles de control integrado de la malaria en México Y América Central. ed. 1. Centro nacional de vigilancia epidemiológica y control de enfermedades. 2004.

10. Realpe M, Hernández C, Agudelo C. Especies del género Bacillus. Morfología macroscópica y microscópica. Biomédica. 2002;22:106-109.

11. Brock M, Martinko J, Madigan M. Biología de los microorganismos. ed 8. Madrid, España. Ed Pearson Education S.A. 2000 p.589-590.

12. Hu X, Fan W, Han B, Liu H, Zheng D, Li Q et al. Complete genome sequence of the mosquitocidal bacterium Bacillus sphaericus C3-41 and comparison with those of closely related Bacillus species. J Bacteriol. 2008;190:2892-2902.

13. Opota O, Charles JF, Warot S, Pauron D, Darboux I. Identification and characterization of the receptor for the Bacillus sphaericus binary toxin in the malaria vector mosquito, Anopheles gambiae. Comp Biochem
Physiol B Biochem Mol Biol. 2008;149:419-427.

14. Nongrados D, Castro J, Mariños C, Laguna A, Ríos R. Eficacia del Bacillus sphaericus 2362 En el control de larvas de Anopheles psudopunctipennis (THEOBALD, 1901) y Culex quinquefasciatus (SAY, 1823) en bioensayos de laboratorio. Rev Peruana Biol. 2002;7:2.

15. Baumann L, Broadwell AH, Baumann P. Sequence analysis of the mosquitocidal toxin genes encoding 51.4- and 41.9-kilodalton proteins from Bacillus sphaericus 2362 and 2297. J Bacteriol. 1988;70:2045-2050.

16. Kalfon A, Charles JF, Bourgouin C, de Barjac H. Sporulation of Bacillus sphaericus 2297: an electron microscope study of crystal-like inclusion biogenesis and toxicity to mosquito larvae. J Gen Microbiol. 1984;130:893-900.

17. Baumann P, Clark MA, Baumann L, Broadwell AH. Bacillus sphaericus as a mosquito pathogen: properties of the organism and its toxins. Microbiol Rev. 1991; 55:425-436.

18. Priest FG, Ebdrup L, Zahner V, Carter PE. Distribution and characterization of mosquitocidal toxin genes in some strains of Bacillus sphaericus. Appl Environ Microbiol. 1997;63:1195-1198.

19. Baumann P, Baumann L, Bowditch RD, Broadwell AH. Cloning of the gene for the larvicidal toxin of Bacillus sphaericus 2362: evidence for a family of related sequences. J Bacteriol. 1987;169:4061-4067.

20. Davidson EW, Oei C, Meyer M, Bieber AL, Hindley J, Berry C. Interaction of the Bacillus sphaericus mosquito larvicidal proteins. Can J Microbiol. 1990; 36:870-878.

21. Pei G, Oliveira CM, Yuan Z, Nielsen-LeRoux C, Silva-Filha MH, Yan J, Regis L. Development of Culex quinquefasciatus resistance to Bacillus sphaericus strain IAB59 needs long term selection pressure. Biological Control. 2007;42:155-160.

22. Rojas J, Mazzarri M, Sojo M, Ysrael G. Evaluación de la efectividad de Bacillus sphaericus cepa 2362 sobre larvas de Anopheles nuñeztovari. Invest. clín; 2001;42:131-146.

23. Maldonado-Blanco M. Control de Aedes aegypti con microorganismos. RESPYN. 2005;6.

24. Mittal PK.. Biolarvicides in vector control: challenges and prospects. J Vector Borne Dis. 2003;40:20-32.

25. Reinert DJ, Carpusca I, Aktories K, Schulz GE. Structure of the Mosquitocidal Toxin from Bacillus sphaericus. J Mol Biol. 2006;357:1226-1236.

26. Humphreys MJ, Berry C. Variants of the Bacillus sphaericus binary toxins: implications for differential toxicity strains. J Invertebr Pathol. 1998;71:184-185.

27. Oei C, Hindley J, Berry C. Binding of purified Bacillus sphaericus binary toxin and its deletion derivatives to Culex quinquefasciatus gut: elucidation of functional binding domains, J Gen Microbiol. 1992;138:1515-1526.

28. Charles JF, Silva-Filha MH, Nielsen-LeRoux C, Humphreys MJ, Berry C. Binding of the 51 and $42 \mathrm{kDa}$ individual components from the Bacillus sphaericus crystal toxin to mosquito larval midgut membranes from Culex and Anopheles sp. FEMS Microbiol Lett. 1997;156:153-159.

29. Broadwell AH, Baumann P. Proteolysis in the gut of mosquito larvae results in further activation of the Bacillus sphaericus toxin, Appl Environ Microbiol. 1987; 53:1333-1337.

30. Baumann P, Clark MA, Baumann L, Broadwell AH. Bacillus sphaericus as a mosquito pathogen: properties of the organism and its toxins, Microbiol. 1991;55: 425-436.

31. Cokmus C, Davidson EW, Cooper K. Electrophysiological effects of Bacillus sphaericus binary toxin on cultured mosquito cells. J Invertebr Pathol. 1997;69:197-204.

32. Charles JF. Ultrastructural midgut events in Culicidae larvae fed with Bacillus sphaericus 2297 spore/crystal complex, Ann Inst Pasteur Microbiol. 1987;138:471-484.

33. SilvaFilha, MH - Peixoto, CA Immunocytochemical localization of the Bacillus sphaericus binary toxin components in Culex quinquefasciatus (Diptera: Culicidae) larvae midgut. 2003;77:138-146.

34. Singh J, Vohra RM, Sahoo DK. Purification and characterization of two extracellular alkaline proteases from a newly isolated obligate alkalophilic Bacillus sphaericus. J Ind Microbiol Biotechnol. 2001;26:387-393. 
35. Singh J, Batra N, Sobti RC. Purification and characterisation of alkaline cellulase produced by a novel isolate, Bacillus sphaericus JS1. J Ind Microbiol Biotechnol. 2004;31:51-56.

36. Singh J. Studies on the production of alkaline protease by a newly isolated Bacillus sphaericus. PhD thesis, Punjab University, handigarh, India. 1998.

37. Singh J, Jasvir RM, Vohra DK. Enhanced production of alkaline proteases by Bacillus sphaericus using fed-batch culture. Process Biochemistry. 2004;39:1093-1101.

38. Almog O, González A, Klein D, Greenblatt HM, Braun S, Shoham G. The $0.93 \mathrm{~A}$ crystal structure of sphericase: a calcium-loaded serine protease from Bacillus sphaericus. J Mol Biol. 2003 3;332:1071-1082.

39. Prabakaran G, Balaraman K. Development of a cost-effective medium for the large scale production of Bacillus thuringiensis var israelensis. Biological Control 2006;36: 288-292.

40. Martins CD, De Aguiar PF, Sérvulo EF. Production of Bacillus sphaericus entomopathogenic biomass using brewery residues. Appl Biochem Biotechnol. 2006;129-132:659-667.

41. Nishiwaki H, Nakashima K, Ishida C, Kawamura T, Matsuda K. Cloning, functional characterization, and mode of action of a novel insecticidal pore-forming toxin, sphaericolysin, produced by Bacillus sphaericus. Appl Environ Microbiol. 2007;73:3404-3411.

42. Tweten RK. Cholesterol-dependent cytolysins, a family of versatile pore-forming toxins. Infect Immun. 2005;73:6199-6209.

43. Poopathi S, Abidha S. Novel fermentation media for production of Bacillus thuringiensis subsp. israelensis. J Econ Entomol. 2003;96:1039-1044.

44. Obeta JA, Okafor N. Medium for the production of primary powder of Bacillus thuringiensis subsp. israelensis. Appl Environ Microbiol. 1984;47:863-867.

45. Saalma H, Foda M, Dulmage HT, Shraby EL. Novel fermentation medium for production of delta-endotoxin from Bacillus thuringiensis. J Invert Pathol. 1983;41:8-19.

46. Kuppusamy M, Balaraman K. Fed-batch fermentation studies with Bacillus thuringiensis $\mathrm{H}-14$ synthesising endotoxin. Indian J Exp Biol. 1991;29:1031-1034.

47. Kumar A, Sra K, Sangodkar U, Sharma VP. Advances in the biocontrol of mosquito vectors utilizing Bacillus sphaericus and Bacillus thuringiensis var. israelensis. Proc Natl Acad Sci India. 2000;1-20.
48. Poopathi S, Charles JF, Nielsen-LeRoux C. Alternative method for preservation of mosquito larvae to study binding mechanisms of Bacillus sphaericus binary toxin. J Invertebr Pathol. 2002;79:132-134.

49. Poopathi S, Kumar KA. Novel fermentation media for production of Bacillus thuringiensis subsp. israelensis. J Econ Entomol. 2003;96:1039-1044.

50. Berti J, Ramírez X, González J, Herrera M. Evaluación de la efectividad de Bacillus sphaericus contra larvas de Anopheles aquasalis Curry (Diptera: Culicidae) en criaderos naturales del estado Sucre, Venezuela. Entomotropica. 2002;17:1-5.

51. Blanco S, Martínez A, Cano O, Tello R, Mendoza I. Introducción del Bacillus sphaericus cepa-2362 (GRISELESF) para el control biológico de vectores maláricos en Guatemala (2000). Rev Cubana MED TROP 2000;52:37-43.

52. Mulla MS, Darwaseh HA, Davidson EW, Dulmage HT, Singer S. Larvicidal activity and field efficacy of Bacillus sphaericus strain mosquito larvae and their safety to non target organism. Mosq News 1984; 44:336-342.

53. Montero G, Díaz M, Marrero A, Castillo F. Resultados de las aplicaciones en pilotaje del biolarvicida Bacillus sphaericus 2362 en criaderos de mosquitos del municipio de Santa Cruz del Norte (Prov. La Habana). Rev. Cuba Med Trop. 1991;43:39-44,

54. Rivera P. Evaluación de la efectividad biolarvicida y residual de Bacillus sphaericus (cepa 2362) para el control de Anopheles albimanus en la costa del lago Xolotlan, Managua, Nicaragua, 1995. Rev Nicar Entomol. 1997; 2:7-14.

55. Villareal LI. Bacillus sphaericus: para el control de vectores de enfermedades tropicales. Rev Latinoam Salud Saneam Ambient $1995 ; 1: 12-4$

56. Organización Panamericana de la Salud (OPS). Empleo inocuo de plaguicidas. Noveno informe del comité de expertos de la OMS en biología de los vectores y lucha antivectorial. Washington, D.C. Serie de Informes Técnicos; No. 720. 1985:26-27.

57. Priest FG. Biological control of mosquitoes and other biting flies by Bacillus sphaericus and Bacillus thuringiensis. J Appl Bacteriol. 1992; 72:357-369.

58. Thiery I, Back C, Barbazan P. Sine'gre G. Application de Bacillus thuringiensis et de B. sphaericus dans la de'moustication et la luttecontre les vecteurs de maladies tropicales. Ann Inst Pasteur Actual. 1996; 7:247-260. 